\title{
On an Inequality of Ostrowski Type via Variant of Pompeiu's Mean Value Theorem
}

\author{
Mehmet Zeki SARIKAYA*, Hüseyin BUDAK \\ Department of Mathematics, Faculty of Science and Arts, Düzce University, Düzce, Turkey \\ *Corresponding author: sarikayamz@gmail.com \\ Received May 17, 2014; Revised June 20, 2014; Accepted June 29, 2014
}

\begin{abstract}
The main of this paper is to establish an Ostrowski type inequality for two variables functions by using a mean value theorem.
\end{abstract}

Keywords: Ostrowski inequality, Pompeiu's mean value theorem

Cite This Article: Mehmet Zeki SARIKAYA, and Hüseyin BUDAK, "On an Inequality of Ostrowski Type via Variant of Pompeiu's Mean Value Theorem." Turkish Journal of Analysis and Number Theory, vol. 2, no. 3 (2014): 80-84. doi: 10.12691/tjant-2-3-5.

\section{Introduction}

The inequality of Ostrowski [7] gives us an estimate for the deviation of the values of a smooth function from its mean value. More precisely, if $f:[a, b] \rightarrow \mathrm{R}$ is a differentiable function with bounded derivative, then

$$
\left|f(x)-\frac{1}{b-a} \int_{a}^{b} f(t) d t\right| \leq\left[\frac{1}{4}+\frac{\left(x-\frac{a+b}{2}\right)^{2}}{(b-a)^{2}}\right](b-a)\left\|f^{\prime}\right\|_{\infty}
$$

for every $x \in[a, b]$. Moreover the constant $1 / 4$ is the best possible. For a differentiable function $f:[a, b] \rightarrow \mathrm{R}, a, b>0$, Dragomir has in [2] proved, using Pompeiu's mean value theorem [6], the following Ostrowski type inequality:

$$
\left|\frac{a+b}{2} \cdot \frac{f(x)}{x}-\frac{1}{b-a} \int_{a}^{b} f(t) d t\right| \leq D(x)\left\|f-\ell f^{\prime}\right\|_{\infty}
$$

where $\ell(t)=t, t \in[a, b]$, and

$$
D(x)=\frac{(b-a)}{|x|}\left[\frac{1}{4}+\frac{\left(x-\frac{a+b}{2}\right)^{2}}{(b-a)^{2}}\right] .
$$

In [4], Pecaric and Ungar proved a general estimate with the $p$-norm, $1<p<\infty$, which will for $p=1$ give the Dragomir [2] result. The interested reader is also referred to $([1,2,3,4,5,8])$ for integral inequalities by using Pompeiu's mean value theorem. In this paper, we establish some new integral inequalities similar to that of the Ostrowski type integral inequality for two variables functions via Pompeiu's mean value theorem.

\section{Main Results}

First we give the following notations used to simplify the details of presentation

$$
\begin{aligned}
& F(u, v)=u v f_{u v}(u, v)-u f_{u}(u, v)-v f_{v}(u, v)+f(u, v) \\
& G(u, v)=v g_{u v}(u, v)-u g_{u}(u, v)-v g_{v}(u, v)+g(u, v),
\end{aligned}
$$

and

$$
\begin{aligned}
& \text { PU }(x, y, p) \\
& \left.=\left(\frac{1-a)^{\frac{1}{p}-1}(d-c) \frac{1}{p}-1}{(1-2 q)(2-q)}+\frac{a^{2-q}-x^{2-q}}{(1-2 q)(1+q)}\right)^{2-q} a^{1+q} x^{1-2 q}\right)^{\frac{1}{q}} \\
& +\left(\frac{c^{2-q}-y^{2-q}}{(1-2 q)(2-q)}+\frac{y^{2-q}-c^{1+q} y^{1-2 q}}{(1-2 q)(1+q)}\right)^{\frac{1}{q}} \\
& +\left(\frac{a^{2-q}-x^{2-q}}{(1-2 q)(2-q)}+\frac{x^{2-q}-a^{1+q} x^{1-2 q}}{(1-2 q)(1+q)}\right)^{\frac{1}{q}} \\
& +\left(\frac{d^{2-q}-y^{2-q}}{(1-2 q)(2-q)}+\frac{y^{2-q}-d^{1+q} y^{1-2 q}}{(1-2 q)(1+q)}\right)^{\frac{1}{q}} \\
& +\left(\frac{b^{2-q}-x^{2-q}}{(1-2 q)(2-q)}+\frac{x^{2-q}-b^{1+q} x^{1-2 q}}{(1-2 q)(1+q)}\right)^{\frac{1}{q}} \\
& +\left(\frac{b^{2-q}-x^{2-q}}{(1-2 q)(2-q)}+\frac{x^{2-q}-b^{1+q} x^{1-2 q}}{(1-2 q)(1+q)}\right)^{\frac{1}{q}} \\
& \left(\frac{c^{2-q}-y^{2-q}}{(1-2 q)(2-q)}+\frac{y^{2-q}-c^{1+q} y^{1-2 q}}{(1-2 q)(1+q)}\right)^{\frac{1}{q}}
\end{aligned}
$$

To prove our theorems, we need the following lemma: 
Lemma 2.1. $f: \Delta=[a, b] \times[c, d] \rightarrow \mathrm{R}$ be an absolutely continuous function such that the partial derivative of order 2 exists for all $(t, s) \in \Delta$ with $0<a<b, 0<c<d$. Then for any $(t, s),(x, y) \in \Delta$, we have

$$
\begin{aligned}
& s t f(x, y)-y t f(x, s)-x s f(t, y)+x y f(t, s) \\
& =x y s t \iint_{t s}^{x y} F(u, v) \frac{d v d u}{u^{2} v^{2}} .
\end{aligned}
$$

Proof. Define $\quad \Psi:\left[\frac{1}{b}, \frac{1}{a}\right] \times\left[\frac{1}{d}, \frac{1}{c}\right] \rightarrow \mathrm{R} \quad$ by $\Psi(t, s):=t s f\left(\frac{1}{t}, \frac{1}{s}\right)$. The function $\Psi$ is continuously differentiable on $\left(\frac{1}{b}, \frac{1}{a}\right) \times\left(\frac{1}{d}, \frac{1}{c}\right)$, and $\quad$ for all $\left(x_{1}, y_{1}\right),\left(x_{2}, y_{2}\right) \in\left[\frac{1}{b}, \frac{1}{a}\right] \times\left[\frac{1}{d}, \frac{1}{c}\right]$, we get

$$
\begin{aligned}
& \Psi\left(x_{1}, y_{1}\right)-\Psi\left(x_{1}, y_{2}\right)-\Psi\left(x_{2}, y_{1}\right)+\Psi\left(x_{2}, y_{2}\right) \\
&= \int_{x_{2}}^{x_{1}} \int_{y_{2}}^{y_{1}} \frac{\partial^{2} \Psi(t, s)}{\partial t \partial s} d s d t \\
&= \int_{x_{2}}^{x_{1}} \int_{y_{2}}^{y_{1}}\left[f\left(\frac{1}{t}, \frac{1}{s}\right)-\frac{1}{s} f_{s}\left(\frac{1}{t}, \frac{1}{s}\right)\right. \\
&\left.-\frac{1}{t} f_{t}\left(\frac{1}{t}, \frac{1}{s}\right)+\frac{1}{t s} f_{t s}\left(\frac{1}{t}, \frac{1}{s}\right)\right] d s d t .
\end{aligned}
$$

Using the change of the variable in last integrals with $u=\frac{1}{t}$ and $v=\frac{1}{s}$, we get

$$
\begin{aligned}
& \Psi\left(x_{1}, y_{1}\right)-\Psi\left(x_{1}, y_{2}\right)-\Psi\left(x_{2}, y_{1}\right)+\Psi\left(x_{2}, y_{2}\right) \\
= & \int_{\frac{1}{x_{2}}}^{\frac{1}{x_{2}}} \frac{1}{y_{2}} \int_{1}^{\frac{1}{y_{1}}}\left[\begin{array}{l}
f(u, v)-v f_{v}(u, v) \\
-u f_{u}(u, v)+u v f_{u v}(u, v)
\end{array}\right] \frac{d v d u}{u^{2} v^{2}} .
\end{aligned}
$$

Denote $x_{1}=\frac{1}{x}, x_{2}=\frac{1}{t}, y_{1}=\frac{1}{y}$ and $y_{2}=\frac{1}{s}$. Then for all $(x, y),(t, s) \in[a, b] \times[c, d]$ from (2.2), we have

$$
\begin{aligned}
& \frac{1}{x y} f(x, y)-\frac{1}{x s} f(x, s)-\frac{1}{t y} f(t, y)+\frac{1}{t s} f(t, s) \\
& =\int_{x y}^{t s} F(u, v) \frac{d v d u}{u^{2} v^{2}}
\end{aligned}
$$

which gives (2.1) and completes the proof.

Theorem $2.1 f: \Delta \rightarrow \mathbf{R}$ be an absolutely continuous function such that the partial derivative of order 2 exists for all $(t, s) \in \Delta$ with $0<a<b, 0<c<d$. Then for $\frac{1}{p}+\frac{1}{q}=1$ with $1 \leq p, q \leq \infty$ any $(t, s),(x, y) \in \Delta$, we have

$$
\begin{aligned}
& \mid \frac{\left(b^{2}-a^{2}\right)\left(d^{2}-c^{2}\right)}{4} \frac{f(x, y)}{x y}-\frac{\left(b^{2}-a^{2}\right)}{2 x} \int_{c}^{d} f(x, s) d s \\
& -\frac{\left(d^{2}-c^{2}\right)}{2 y} \int_{a}^{b} f(t, y) d t+\int_{a c}^{b d} f(t, s) d s d t \mid \\
& \leq P U(x, y, p)\left\|l_{1} f_{u v}-r l_{2} f_{u}-l_{3} f_{v}+f\right\|_{p}
\end{aligned}
$$

where $l_{1}(x, y)=x y, l_{2}(x, \cdot)=x$ and $l_{3}(\cdot, y)=y$ for all $(x, y) \in \Delta$.

Proof From Lemma 2.1, we have

$$
\begin{aligned}
& s t f(x, y)-y t f(x, s)-x s f(t, y)+x y f(t, s) \\
& =x y s t \iint_{t s}^{x y} F(u, v) \frac{d v d u}{u^{2} v^{2}} .
\end{aligned}
$$

Integrating with respect to $(t, s)$ on $[a, b] \times[c, d]$ and dividing by $x y$, we get

$$
\begin{aligned}
& \frac{\left(b^{2}-a^{2}\right)\left(d^{2}-c^{2}\right)}{4} \frac{f(x, y)}{x y}-\frac{\left(b^{2}-a^{2}\right)}{2 x} \int_{c}^{d} f(x, s) d s \\
& -\frac{\left(d^{2}-c^{2}\right)}{2 y} \int_{a}^{b} f(t, y) d t+\int_{a c}^{b d} f(t, s) d s d t \\
= & \int_{a c}^{b d} s t\left[\iint_{t s}^{x y} F(u, v) \frac{d v d u}{u^{2} v^{2}}\right] d s d t
\end{aligned}
$$

and therefore

$$
\begin{aligned}
& \mid \frac{\left(b^{2}-a^{2}\right)\left(d^{2}-c^{2}\right)}{4} \frac{f(x, y)}{x y}-\frac{\left(b^{2}-a^{2}\right)}{2 x} \int_{c}^{d} f(x, s) d s \\
& -\frac{\left(d^{2}-c^{2}\right)}{2 y} \int_{a}^{b} f(t, y) d t+\int_{a c}^{b d} f(t, s) d s d t \mid \\
\leq & \int_{a c}^{b d}\left|\int_{t s}^{x y}\right| F(u, v)\left|\frac{t s d v d u}{u^{2} v^{2}}\right| d s d t \\
= & \int_{a c}^{x y} \int\left|\int_{x y}^{t s}\right| F(u, v)\left|\frac{t s d v d u}{u^{2} v^{2}}\right| d s d t
\end{aligned}
$$$$
\begin{aligned}
& +\int_{a y}^{x d}\left|\int_{\mid x y}^{t s}\right| \int_{x y}|F(u, v)| \frac{t s d v d u}{u^{2} v^{2}} \mid d s d t \\
& +\int_{x c}^{b y}\left|\int_{x y}^{t s}\right| F(u, v)\left|\frac{t s d v d u}{u^{2} v^{2}}\right| d s d t
\end{aligned}
$$$$
+\int_{x y}^{b d} \int_{x y}^{t} \iint_{x y}^{t s}|F(u, v)| \frac{t s d v d u}{u^{2} v^{2}} \mid d s d t .
$$

Firstly, we will consider the case $1<p, q<\infty$. By using Hölder's inequality, the sum in the last line (2.5) is

$$
\begin{gathered}
\leq\left(\int_{a c}^{x y}\left(\left.\int_{t s}^{x y}\left|\int_{s}\right| F(u, v)\right|^{p} d v d u\right) d s d t\right)^{\frac{1}{p}} \\
\quad \times\left(\iint_{a c}^{x y}\left(\iint_{t s}^{x y} \frac{t^{q} s^{q} d v d u}{u^{2 q} v^{2 q}}\right) d s d t\right)^{\frac{1}{q}}
\end{gathered}
$$




$$
\begin{aligned}
& +\left(\int_{a y}^{x d}\left(\int_{t}^{x s}|F(u, v)|^{p} d v d u\right) d s d t\right)^{\frac{1}{p}} \\
& \times\left(\int_{a}^{x d}\left(\int_{y}^{x s} \int_{t y}^{t^{q} s^{q} d v d u} u^{2 q} v^{2 q}\right) d s d t\right)^{\frac{1}{q}} \\
& +\left(\int_{x c}^{b} \int_{x s}^{y}\left(\int_{x s}^{t y}|F(u, v)|^{p} d v d u\right) d s d t\right)^{\frac{1}{p}} \\
& \times\left(\int_{x c}^{b y}\left(\int_{x s}^{t y} \int_{t^{q}} \frac{t^{q}{ }^{q} d v d u}{u^{2 q} v^{2 q}}\right) d s d t\right)^{\frac{1}{q}} \\
& +\left(\int_{x y}^{b d}\left(\int_{x y}^{t} \int^{s}|F(u, v)|^{p} d v d u\right) d s d t\right)^{\frac{1}{p}} \\
& \times\left(\int_{x y}^{b d}\left(\int_{x y}^{t} \int_{u^{2}}^{t^{q} s^{q} d v d u} \frac{u^{2 q}}{u^{2 q}}\right) d s d t\right)^{\frac{1}{q}} \\
& \leq\left(\int_{a c}^{b d}\left(\int_{a c}^{b d}|F(u, v)|^{p} d v d u\right) d s d t\right)^{\frac{1}{p}} \\
& =\left(\frac{a^{2-q}-x^{2-q}}{(1-2 q)(2-q)}+\frac{x^{2-q}-a^{1+q} x^{1-2 q}}{(1-2 q)(1+q)}\right)^{\frac{1}{q}} \\
& \left(\frac{c^{2-q}-y^{2-q}}{(1-2 q)(2-q)}+\frac{y^{2-q}-c^{1+q} y^{1-2 q}}{(1-2 q)(1+q)}\right)^{\frac{1}{q}} \\
& +\left(\frac{a^{2-q}-x^{2-q}}{(1-2 q)(2-q)}+\frac{x^{2-q}-a^{1+q} x^{1-2 q}}{(1-2 q)(1+q)}\right)^{\frac{1}{q}} \\
& \left(\frac{d^{2-q}-y^{2-q}}{(1-2 q)(2-q)}+\frac{y^{2-q}-d^{1+q} y^{1-2 q}}{(1-2 q)(1+q)}\right)^{\frac{1}{q}} \\
& +\left(\frac{b^{2-q}-x^{2-q}}{(1-2 q)(2-q)}+\frac{x^{2-q}-b^{1+q} x^{1-2 q}}{(1-2 q)(1+q)}\right)^{\frac{1}{q}} \\
& \left(\frac{c^{2-q}-y^{2-q}}{(1-2 q)(2-q)}+\frac{y^{2-q}-c^{1+q} y^{1-2 q}}{(1-2 q)(1+q)}\right)^{\frac{1}{q}} \\
& +\left(\frac{b^{2-q}-x^{2-q}}{(1-2 q)(2-q)}+\frac{x^{2-q}-b^{1+q} x^{1-2 q}}{(1-2 q)(1+q)}\right)^{\frac{1}{q}} \\
& \left(\frac{d^{2-q}-y^{2-q}}{(1-2 q)(2-q)}+\frac{y^{2-q}-d^{1+q} y^{1-2 q}}{(1-2 q)(1+q)}\right)^{\frac{1}{q}} \text {. }
\end{aligned}
$$$$
\left\{\begin{array}{l}
\left(\int_{a c}^{x y}\left(\int_{t}^{x y} \int_{s}^{t} \frac{t^{q} s^{q} d v d u}{u^{2 q} v^{2 q}}\right) d s d t\right)^{\frac{1}{q}} \\
+\left(\int_{a y}^{x d}\left(\int_{t y}^{x s} \frac{t^{q} s^{q} d v d u}{u^{2 q} v^{2 q}}\right) d s d t\right)^{\frac{1}{q}} \\
+\left(\int _ { x c } ^ { b y } \left(\int_{x c}^{t y} \int_{x s}^{\left.\left.\frac{t^{q} s^{q} d v d u}{u^{2 q} v^{2 q}}\right) d s d t\right)^{\frac{1}{q}}}\right.\right. \\
+\left(\int_{x y}^{b d}\left(\int_{x y}^{t s} \frac{t^{q} s^{q} d v d u}{u^{2 q} v^{2 q}}\right) d s d t\right)^{\frac{1}{q}}
\end{array}\right\} .
$$

The first factor in (2.6) equals

$$
\begin{aligned}
& \left(\int_{a c}^{b d}\left(\int_{a c}^{b d}|F(u, v)|^{p} d v d u\right) d s d t\right)^{\frac{1}{p}} \\
= & (b-a)^{\frac{1}{p}}(d-c)^{\frac{1}{p}}\left\|l_{1} f_{u v}-r l_{2} f_{u}-l_{3} f_{v}+f\right\|_{p} .
\end{aligned}
$$

and for the second factor, for $p, q \neq 2$, we get

$$
\left\{\begin{array}{c}
\left(\int_{a c}^{x y}\left(\int_{t s}^{x y} \int_{t^{q} s^{q} d v d u}^{u^{2 q} v^{2 q}}\right) d s d t\right)^{\frac{1}{q}}+\left(\int_{a y}^{x d}\left(\int_{t y}^{x s} \int_{t y}^{t^{q} s^{q} d v d u} \frac{u^{2 q} v^{2 q}}{u^{2}} d s d t\right)^{\frac{1}{q}}\right. \\
+\left(\int_{x c}^{b y} \int\left(\int_{x s}^{t y} \frac{t^{q} s^{q} d v d u}{u^{2 q} v^{2 q}}\right) d s d t\right)^{\frac{1}{q}}+\left(\int_{x y}^{b d} \int\left(\int_{x y}^{t s} \frac{t^{q} s^{q} d v d u}{u^{2 q} v^{2 q}}\right) d s d t\right)^{\frac{1}{q}}
\end{array}\right\}
$$

For $p=q=2$, instead of (2.8), we obtain

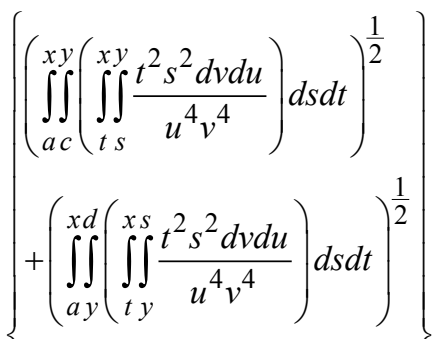

$$
\begin{aligned}
& +\left(\int_{x c}^{b y}\left(\int_{x s}^{t} \int_{u^{2}}^{y} \frac{t^{2} s^{2} d v d u}{u^{4}{ }^{4}}\right) d s d t\right)^{\frac{1}{2}} \\
& \left.+\left(\int_{x y}^{b d}\left(\int_{x y}^{t} \int_{u^{4} v^{4}}^{t^{2} s^{2} d v d u}\right)^{4}\right) d s d t\right)^{\frac{1}{2}} \\
& =\frac{1}{9}\left[\left(\ln \left(\frac{x}{a}\right)^{3}+\frac{a^{3}}{x^{3}}-1\right)^{\frac{1}{2}}+\left(\ln \left(\frac{x}{b}\right)^{3}+\frac{b^{3}}{x^{3}}-1\right)^{\frac{1}{2}}\right] \\
& \times\left[\left(\ln \left(\frac{y}{c}\right)^{3}+\frac{c^{3}}{y^{3}}-1\right)^{\frac{1}{2}}+\left(\ln \left(\frac{y}{d}\right)^{3}+\frac{d^{3}}{y^{3}}-1\right)^{\frac{1}{2}}\right]
\end{aligned}
$$

which is easily shown to be equal to the limit of the right hand side of (2.8) for $q \rightarrow 2$, i.e.

$$
\lim _{q \rightarrow 2} P U(x, y, p)=\frac{1}{9(b-a)^{\frac{1}{q}}(d-c)^{\frac{1}{q}}}
$$




$$
\times\left[\begin{array}{l}
\left(\ln \left(\frac{x}{a}\right)^{3}+\frac{a^{3}}{x^{3}}-1\right)^{\frac{1}{2}}\left(\ln \left(\frac{x}{b}\right)^{3}+\frac{b^{3}}{x^{3}}-1\right)^{\frac{1}{2}} \\
+\left(\ln \left(\frac{y}{c}\right)^{3}+\frac{c^{3}}{y^{3}}-1\right)^{\frac{1}{2}}\left(\ln \left(\frac{y}{d}\right)^{3}+\frac{d^{3}}{y^{3}}-1\right)^{\frac{1}{2}}
\end{array}\right] .
$$

Now, consider the case $p=\infty, q=1$. Then, the last line in $(2.5)$ is

$$
\begin{aligned}
& \left.\leq \sup _{(u, v) \in[a, b] \times[c, d]} \mid F(u, v)\right) \mid \\
& \times\left\{\int_{a c}^{x y}\left(\int_{t s}^{x y} \frac{t s d v d u}{u^{2} v^{2}}\right) d s d t d+\int_{a y}^{x d}\left(\int_{t y}^{x s} \frac{t s d v d u}{u^{2} v^{2}}\right) d s d t\right. \\
& \left.\left.+\int_{x c}^{b y}\left(\int_{x s}^{t y} \frac{t s d v d u}{u^{2} v^{2}}\right) d s d t+\int_{x y}^{b d} \int_{x y}^{t s} \int_{x y}^{s} \frac{t s d v d u}{u^{2} v^{2}}\right) d s d t\right\} \\
& =\left\|l_{1} f_{u v}-l_{2} f_{u}-l_{3} f_{v}+f\right\|_{\infty} \\
& \left(\frac{a^{2}+b^{2}}{2 x}+x-a-b\right)\left(\frac{c^{2}+d^{2}}{2 y}+y-c-d\right) .
\end{aligned}
$$

Putting (2.10) into (2.5) and dividing by $(b-a)(d-c)$ gives

$$
\begin{aligned}
& \left|\begin{array}{l}
\frac{(a+b)(c+d)}{4} \frac{f(x, y)}{x y}-\frac{a+b}{2 x\left(d^{2} c^{2}\right)} \int_{c}^{d} f(x, s) d s \\
-\frac{c+d}{2 y\left(b^{2}-a^{2}\right)} \int_{a}^{b} f(t, y) d t \\
+\frac{1}{\left(b^{2}-a^{2}\right)\left(d^{2} c^{2}\right)} \int_{a c}^{b d} f(t, s) d s d t
\end{array}\right| \\
& \leq \frac{1}{(b-a)(d-c)}\left\|l_{1} f_{u v}-l_{2} f_{u}-l_{3} f_{v}+f\right\|_{\infty} \\
& \times\left(\frac{a^{2}+b^{2}}{2 x}+x-a-b\right)\left(\frac{c^{2}+d^{2}}{2 y}+y-c-d\right) .
\end{aligned}
$$

Finally, we consider the case $p=1, q=\infty$. then, the last line of (2.5) is

$$
\begin{aligned}
\leq & \int_{a c}^{x y} \int\left(\int_{t}^{x y}|F(u, v)| \max _{\substack{(u, v) \in[t, x] \times[s, y] \\
(t, s) \in[a, x] \times[c, y]}} \frac{t s}{u^{2} v^{2}} d v d u\right) d s d t \\
& +\int_{a y}^{x d}\left(\int_{t}^{t} \int_{x y}^{t}|F(u, v)| \underset{\substack{(u, v) \in[t, x] \times[y, s] \\
(t, s) \in[a, x] \times[c, y]}}{\max u^{2} v^{2}} d v d u\right) d s d t
\end{aligned}
$$$$
+\int_{x c}^{b y} \int\left(\int_{x s}^{t y}|F(u, v)| \max _{\substack{(u, v) \in[x, t] \times[s, y] \\(t, s) \in[a, x] \times[c, y]}} \frac{t s}{u^{2} v^{2}} d v d u\right) d s d t
$$

$$
+\int_{x y}^{b d}\left(\int_{x y}^{t s}|F(u, v)| \max _{\substack{(u, v) \in[x, t] \times[y, s] \\(t, s) \in[a, x] \times[c, y]}} \frac{t s}{u^{2} v^{2}} d v d u\right) d s d t
$$

$$
\begin{aligned}
& \left.\leq \int_{a c}^{b d}\left(\int_{a c}^{b d} \mid F(u, v)\right) \mid d v d u\right) d s d t \cdot\left(\frac{1}{a}+\frac{b}{x^{2}}\right)\left(\frac{1}{c}+\frac{d}{y^{2}}\right) \\
& =(b-a)(d-c)\left(\frac{1}{a}+\frac{b}{x^{2}}\right)\left(\frac{1}{c}+\frac{d}{y^{2}}\right) \\
& \times\left\|l_{1} f_{u v}-l_{2} f_{u}-l_{3} f_{v}+f\right\|_{1}
\end{aligned}
$$

Appending (2.11) to (2.5) and dividing by $(b-a)(d-c)$ gives

$$
\begin{aligned}
& \left|\begin{array}{l}
\frac{(a+b)(c+d)}{4} \frac{f(x, y)}{x y} \\
-\frac{a+b}{2 x\left(d^{2}-c^{2}\right)} \int_{c}^{d} f(x, s) d s \\
-\frac{c+d}{2 y\left(b^{2}-a^{2}\right)} \int_{a}^{b} f(t, y) d t \\
+\frac{1}{(b-a)(d-c)} \int_{a c}^{b d} f(t, s) d s d t
\end{array}\right| \\
& \leq\left(\frac{1}{a}+\frac{b}{x^{2}}\right)\left(\frac{1}{c}+\frac{d}{y^{2}}\right)\left\|l_{1} f_{u v}-l_{2} f_{u}-l_{3} f_{v}+f\right\|_{1} .
\end{aligned}
$$

It is not too difficult to show that

$$
\lim _{p \rightarrow 1} P U(x, y, p)=\left(\frac{1}{a}+\frac{b}{x^{2}}\right)\left(\frac{1}{c}+\frac{d}{y^{2}}\right)
$$

so (2.12) proves formula (2.3) for $p=1, q=\infty$, proving the theorem.

heorem $2.2 f: \Delta \rightarrow \mathrm{R}$ be an absolutely continuous function such that the partial derivative of order 2 exists for all $(t, s) \in \Delta$ with $0<a<b, 0<c<d$, and let $w: \Delta \rightarrow \mathrm{R}$ be a nonnegative integrable function. Then for $\frac{1}{p}+\frac{1}{q}=1$ with $1 \leq p, q \leq \infty$ any $(t, s),(x, y) \in \Delta$, we have

$$
\begin{aligned}
& \mid \frac{f(x, y)}{x y} \int_{a c}^{b d} w(t, s) d s d t-\frac{1}{x} \int_{a c}^{b d} \int_{a c} w(t, s) f(x, s) d s d t \\
& -\frac{1}{y} \int_{a c}^{b d} w(t, s) f(t, y) d t+\int_{a c}^{b d} w(t, s) f(t, s) d s d t \mid \\
& \leq \frac{(b-a)^{\frac{1}{p}}(d-c)^{\frac{1}{p}}}{(1-2 q)^{\frac{1}{q}}}\left\|l_{1} f_{u v}-r l_{2} f_{u}-l_{3} f_{v}+f\right\|_{p} \\
& \times\left\{\left(\int_{a c}^{x y}\left[x^{1-2 q} t^{q}-t^{1-q}\right]\left[y^{1-2 q} s^{q}-s^{1-q}\right] w^{q}(t, s) d s d t\right)^{\frac{1}{q}}\right. \\
& +\left(\int_{a y}^{x d}\left[x^{1-2 q} t^{q}-t^{1-q}\right]\left[s^{1-q}-y^{1-2 q} s^{q}\right] w^{q}(t, s) d s d t\right)^{\frac{1}{q}} \\
& +\left(\int_{a y}^{b y}\left[t_{x c}^{1-q}-x^{1-2 q} t^{q}\right]\left[y^{1-2 q} s^{q}-s^{1-q}\right] w^{q}(t, s) d s d t\right)^{\frac{1}{q}}
\end{aligned}
$$




$$
+\left(\int_{x y}^{b d} \times\left[s^{1-q}-y^{1-2 q} s^{q}\right] w^{q}(t, s) d s d t\right)^{\frac{1}{q}} d .
$$

Proof Multiplying (2.4) by $\frac{w(t, s)}{x y}$ and integrating with respect to $(t, s)$ on $[a, b] \times[c, d]$, we have

$$
\begin{aligned}
& \frac{f(x, y)}{x y} \int_{a c}^{b d} \int_{c}^{d} w(t, s) d s d t-\frac{1}{x} \int_{a c}^{b d} \int_{c}^{d} w(t, s) f(x, s) d s d t \\
& -\frac{1}{y} \int_{a c}^{b d} \int_{a} w(t, s) f(t, y) d t+\iint_{a c}^{b d} w(t, s) f(t, s) d s d t \\
= & \int_{a c}^{b d} s t w(t, s)\left[\int_{t s}^{x y}[F(u, v)] \frac{d v d u}{u^{2} v^{2}}\right] d s d t
\end{aligned}
$$

and as in the proof of Theorem 2.1, we get

$$
\begin{aligned}
& \frac{f(x, y)}{x y} \int_{a c}^{b d} w(t, s) d s d t-\frac{1}{x} \int_{a c}^{b d} \int_{a c}^{d} w(t, s) f(x, s) d s d t \\
& -\frac{1}{y} \int_{a c}^{b d} w(t, s) f(t, y) d t+\int_{a c}^{b d} \int_{c}^{d} w(t, s) f(t, s) d s d t \\
& \leq \int_{a c}^{b d}\left|\int_{\mid}^{x} \int_{t s}^{y}\right| F(u, v)\left|\frac{t s w(t, s) d v d u}{u^{2} v^{2}}\right| d s d t \\
& \left.=\int_{a c}^{x y} \int_{t}^{x y} \iint_{t}^{x}|F(u, v)| \frac{t s w(t, s) d v d u}{u^{2} v^{2}}\right) d s d t \\
& +\int_{a y}^{x d} \int_{y}^{x}\left(\int_{t y}^{x s}|F(u, v)| \frac{t s w(t, s) d v d u}{u^{2} v^{2}}\right) d s d t \\
& +\int_{x c}^{b y} \int\left(\int_{x s}^{t} \int|F(u, v)| \frac{t s w(t, s) d v d u}{u^{2} v^{2}}\right) d s d t \\
& +\int_{x y}^{b d} \int\left(\int_{x y}^{t} \int|F(u, v)| \frac{t s w(t, s) d v d u}{u^{2} v^{2}}\right) d s d t \\
& \leq\left[\int_{a c}^{x y}\left(\int_{t s}^{x y}|F(u, v)|^{p} d v d u\right) d s d t\right]^{\frac{1}{p}} \\
& {\left[\int_{a c}^{x y}\left(\int_{t}^{x y} \int_{s}^{t^{q} s^{q} w^{q}(t, s) d v d u} \frac{u^{2 q} v^{2 q}}{d s d t}\right]^{\frac{1}{q}}\right.} \\
& +\left[\int_{a}^{x d} \int_{y}\left(\int_{t}^{x s}|F(u, v)|^{p} d v d u\right) d s d t\right]^{\frac{1}{p}}
\end{aligned}
$$

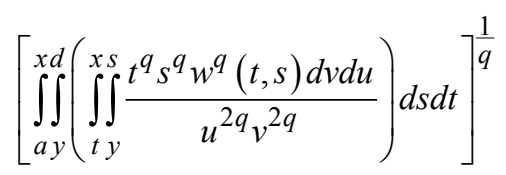

$$
\begin{aligned}
& +\left[\int_{x c}^{b} \int_{x s}^{y}\left(\int_{x}^{t y}|F(u, v)|^{p} d v d u\right) d s d t\right]^{\frac{1}{p}} \\
& {\left[\int_{x c}^{b} \int\left(\int_{x s}^{t} \int_{s}^{y} \frac{t^{q} s^{q} w^{q}(t, s) d v d u}{u^{2 q} v^{2 q}}\right) d s d t\right]^{\frac{1}{q}}} \\
& \left.+\left[\left.\int_{x y}^{b d}\left(\int_{x y}^{t} \int \mid F(u, v)\right)\right|^{p} d v d u\right) d s d t\right]^{\frac{1}{p}} \\
& {\left[\int_{x y}^{b} \int_{x y}^{d}\left(\int_{x y}^{t} \int_{u^{2 q} v^{2 q}}^{t^{q} s^{q} w^{q}(t, s) d v d u}\right) d s d t\right]^{\frac{1}{q}}} \\
& \leq\left[\int_{a c}^{b d}\left(\int_{a c}^{b d}|F(u, v)|^{p} d v d u\right) d s d t\right]^{\frac{1}{p}} \\
& \left\{\left[\iint_{a c}^{x y}\left(\int_{t}^{x y} \frac{t^{q} s^{q} w^{q}(t, s) d v d u}{u^{2 q} v^{2 q}}\right) d s d t\right]^{\frac{1}{q}}\right.
\end{aligned}
$$

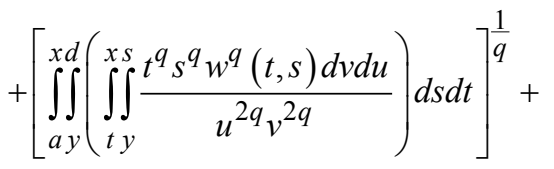

$$
\begin{aligned}
& {\left[\int_{x c}^{b y}\left(\int_{x s}^{t} \int_{x}^{y} \frac{t^{q} s^{q} w^{q}(t, s) d v d u}{u^{2 q} v^{2 q}}\right) d s d t\right]^{\frac{1}{q}}}
\end{aligned}
$$

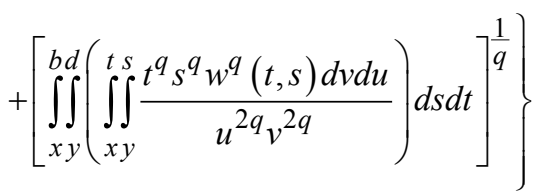

which gives (2.13).

\section{References}

[1] A. M. Acu, A. Babos and F. D. Sofonea, The mean value theorems and inequalities of Ostrowski type. Sci. Stud. Res. Ser. Math. Inform. 21 (2011), no. 1, 5-16.

[2] S.S. Dragomir, An inequality of Ostrowski type via Pompeiu's mean value theorem, J. of Inequal. in Pure and Appl. Math., 6(3) (2005), Art. 83.

[3] I. Muntean, Extensions of some mean value theorems, BabesBolyai University, Faculty of Mathematics, Research Seminars on Mathematical Analysis, Preprint Nr. 7, 1991, 7-24.

[4] P.P Pecaric and S. Ungar, On an inequality of Ostrowski type, J. of Inequal. in Pure and Appl. Math., 7(4) (2006), Art. 151.

[5] E. C. Popa, An inequality of Ostrowski type via a mean value theorem, General Mathematics Vol. 15, No. 1, 2007, 93-100.

[6] D. Pompeiu, Sur une proposition analogue au théorème des accroissements finis, Mathematica (Cluj, Romania), 22 (1946), 143-146.

[7] A. Ostrowski, Uber die Absolutabweichung einer differentierbaren Funktionen von ihrem Integralmittelwert, Comment. Math. Helv., 10(1938), 226-227.

[8] F. Ahmad, N. A. Mir and M.Z. Sarikaya, An inequality of Ostrowski type via variant of Pompeiu's mean value theorem, J. Basic. Appl. Sci. Res., 4(4)204-211, 2014. 